\title{
VIII Jornadas de Ciencia y Tecnología
}

\section{Jornadas Nacionales de Becarios y Tesistas}

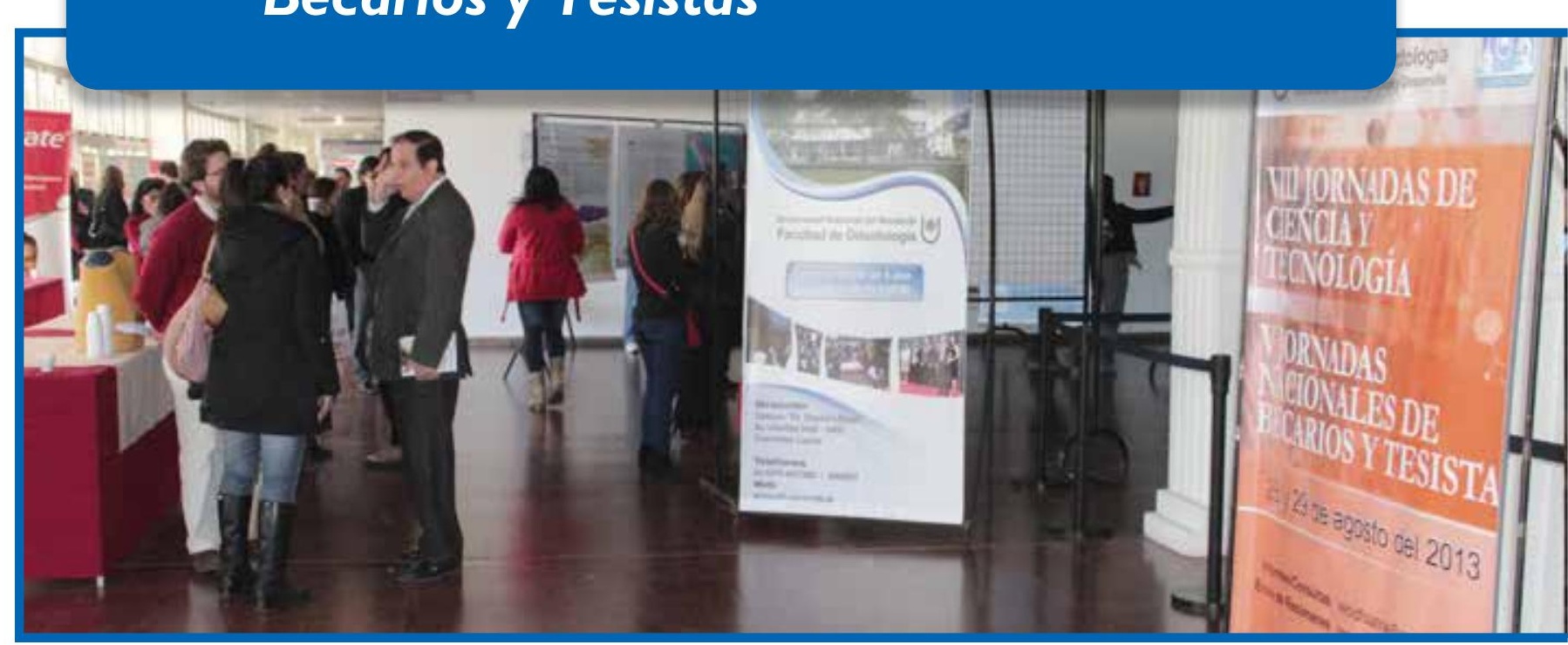

Os días 28 y 29 agosto se realizaron las VIII Lornadas de Ciencia y Tecnología y la V Jornadas Nacionales de Becarios y Tesistas" organizadas por la Secretaría de Investigación y Desarrollo de la Facultad de Odontología, a cargo de la Prof. Mgter. Susana Finten.

En acto de apertura estuvieron presentes el Sr. Decano de la Facultad, Prof. Roque Oscar Rosende; la Sra. Vice Decana, Prof. María Mercedes González; la Sra. Secretaria Gral. de Ciencia y Técnica de la UNNE, Dra. Silvia Mazza; la Sra. Secretaria de Investigación y Desarrollo de la Facultad de Odontología, Prof. Susana Finten.

Acompañaron en este acto Decanos de otras unidades académicas, Secretarios Generales y autoridades de la facultad, docentes investigadores, becarios de pregrado y posgrado, personal no docente y estudiantes de la carrera.

En primer lugar, la Prof. Susana Finten realizó la apertura con una breve reseña sobre la evolución de las actividades de investigación en la facultad Destacó que “...desde el año 2010, se continúa con el apoyo de estas acciones, para seguir avanzando con ideas innovadoras, formulando trabajos de investigación, que se anhela culminen con la concreción de patentes".

Finalmente, para cerrar el acto, el Decano de la facultad hizo uso de la palabra aclarando que este espacio pretende "generar una actitud de crítica, de creación, emprendimiento y de generación de nuevos conocimientos.

Continuando con la programación, se realizaron Conferencias: "Microscopía con focal, aplicaciones y alcances de esta tecnología." doctor Carlos Mas auspiciado por Bioanalítica Argentina S.A. y la dra Silvia Mazza dicto una conferencia sobre Criterios de evaluación del personal en Ciencia y Técnica.

Seguidamente se realizaron las exposiciónes orales de protocolos y trabajos de investigación finalizados a cargo de los docentes investigadores. 


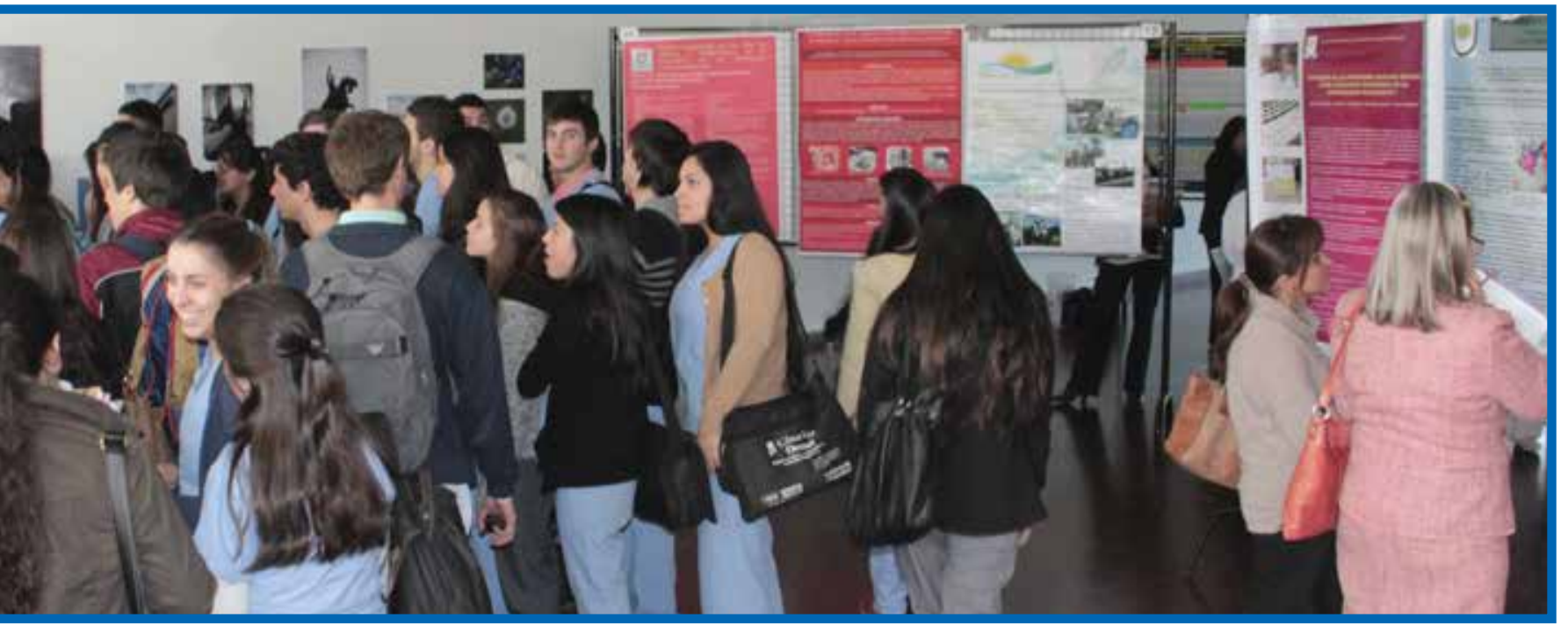

\section{Trabajos Presentados}

"Estudio In Vitro de la Microfiltracion utilizando un sistema de adhesion convencional monoenvase". Autores: Aguirre Grabre de Prieto Alicia, Zamudio Maria E.

"Efectos de los contactos mediotrusivos en la articulacion temporo mandibular". Autores: Altamirano Ricardo H., Collante Carmen, Álvarez Omar.

"Estudio comparativo In Vitro de la fuerza de union sustrato-sistema adhesivo -resina reforzada según tratamiento recibido por el esmalte dentario". Autores: Álvarez Nilda M., Zamudio Maria E.

"Determinacion de la estabilidad del color y resistencias a la compresion y flexion de dos materiales utilizados para la confeccion de coronas provisorias". Autores: Christiani Juan J., Zamudio Maria E., Devecchi José R., Rocha María T., Avalos Llano Karina.

"Ubicacion del hueso hioides en pacientes con convexidad facial reducida". Autores: Collante de Benítez Carmen I., Latyn de Enz Karina, Palczikaowski Lorena.

"Determinacion del genero a traves de la Odontometria". Autores: Rivarola José R., Ariasgago Olga L.

"Determinacion inmunohistoquimica de ghrelina en odontolblastos de piezas dentarias permanentes". Autores: Segovia, Silvia M., Valdovinos Zaputovich Bertha, Alsina Ángel E., Enz Natalie.

"Enfermedad Periodontal: Microorganismos Periodontopatógenos Relacionados". Autores: Silvia Ortega, Javier Monzón, Cynthya Sin, Karina Soto Mayol, María R. Britos, Olga Miriam Vasek.

"Medicación intraconducto utilizando gel de aloe vera y acondicionamiento final del conducto radicular".

Autora: Finten Susana B.

"Evaluación clínica de diferentes ionómeros vítreos de restauración con amalgama en molares y premolares permanentes, como alternativa rehabilitadora en atención primaria de salud". Autores: Mandri María N., Zamudio Maria $E$. 
REVISTA FACULTAD DE ODONTOLOGÍA

ISSN No 1668-7280 - Vol. VI № 1 - 2013

80

\section{NOTICIAS}

Como así también la presentación de protocolos o avances de los proyectos de investigación de becarios de la unidad académica y de otras facultades de odontología del país.

\section{Modalidad Póster}

I. "Estudio Morfométrico del Primer Molar Superior Permanente". Autores: Aquino Carlos J., Bessone Gabriela G., Ruiz Díaz de Centeno E.

2. "Fosfatasa alcalina salival como medio de diagnostico de Periimplantitis”. Autores: Azzi Elías J., Acuña Miguel J., Juárez Rolando P.

3. "Prevalencia de signos y síntomas temporomandibulares en pacientes adultos mayores que concurren a la Cátedra de Cirugía II Dentomaxilar". Autores: Bernachea Luciana, López Vallejos María J., Rosende Roque $O$.

4. "Prevalencia de lesiones y alteraciones clínicas en las áreas para protésicas de los pacientes portadores de prótesis parcial removible". Autores: Brunás Rosina L., Huber Laura B., Altamirano María M., Rosende Roque $O$.

5. "Determinación de la prevalencia del tubérculo de Carabelli, en adolescentes". Autores: Casullo Eduardo H., Bessone Gabriela G.

6. "Estudio Epidemiológico de la presencia del HPV en patologías estomatológicas”. Autores: De Langhe María V., González María M.

7. "Consulta odontológica en individuos adultos de la Ciudad de Corrientes en relación al nivel socioeconómico". Autora: Dho María S.

8. "Comparación clínica de dos selladores de fosas y fisuras aplicados en pacientes normales y con discapacidad motora." Autores: Encina Tutuy Alejandro J., Martínez Sandra E., Vila Vilma $G$.

9. "Estudio comparativo de diversas técnicas de diagnóstico de caries dental en dentición permanente." Autores: Falcón Diana E, Valdovinos Zaputovich Bertha M, Discacciati de Lértora María S.

10. “Determinación de la acción térmica del mate como factor predisponente del cáncer bucal." Autores: Fernández Estefanía R., González María M.

II. EV "Determinación de la presencia de
Queilitis comisurales en los pacientes que concurren Cátedra de Cirugía I. Técnicas quirúrgicas y anestésicas." Autores: Ferreyra María A., Rosende Roque O., Fernández Víctor $R$.

12. "Efectividad Antimicrobiana entre propoleos de la región de Corrientes y de la región de Cuyo ante el estreptococos mutans." Autores: Fleita Romina A., Rosende Roque O., Vaculik Patricia A.

I3. "Relación entre al estado nutricional y prevalencia de hipomineralización incisiva molar en niños que concurren en Cátedra de Odontopediatría de la FOUNNE." Autores: Kunz María V., Cardoso María L., Discaciatti de Lértora María S.

14. "Perfil Inmunohistoquimico del ameloblastoma de valor pronóstico y predictivo". Autores: Fortín Pedro; Briend Maria S., Morales Sergio $D$.

15. "Terapéutica farmacológica en analgesia dental utilizada por los pobladores del Iberá." Autores: Gauna Rita S., Morales Sergio D., Karaben Viviana $E$.

16. "Caracterización de la apariencia visual de las superficies pulidas de resinas acrílicas de termo curado mediante procesamiento digital de imagen." Autores: Gómez Valeria J., Huber Laura B., Zamudio María E., Altamirano Maria $M$.

17. "Concentración de fosfatasa alcalina salival en pacientes con periodontitis severa." Autores: Harvey Alina, Acuña Miguel J., Celia Armando, Cuzziol Fernando, Juárez Rolando $P$.

18. "Rol de la inmunoglobulina a salival como marcador biológico de la caries dental." Autores: Hormaechea María l., Martínez Sandra E., Vila Vilma G., Juárez Rolando P.

19. "Estudio retrospectivo de las citologías recibidas en el servicio de Anatomía Patológica de la facultad de Odontología de la UNNE durante el período 2007- 2013." Autores: Ibarra Silvana N., Montenegro María de las A., Almirón María S.

20. "Péptidos con actividad remineralizante generados por bacterias lácticas autóctonas." Autores: Koch Karina E., Vasek Olga M., Ortega Silvia $M$.

2I. "Concentración de fosfatasa alcalina 


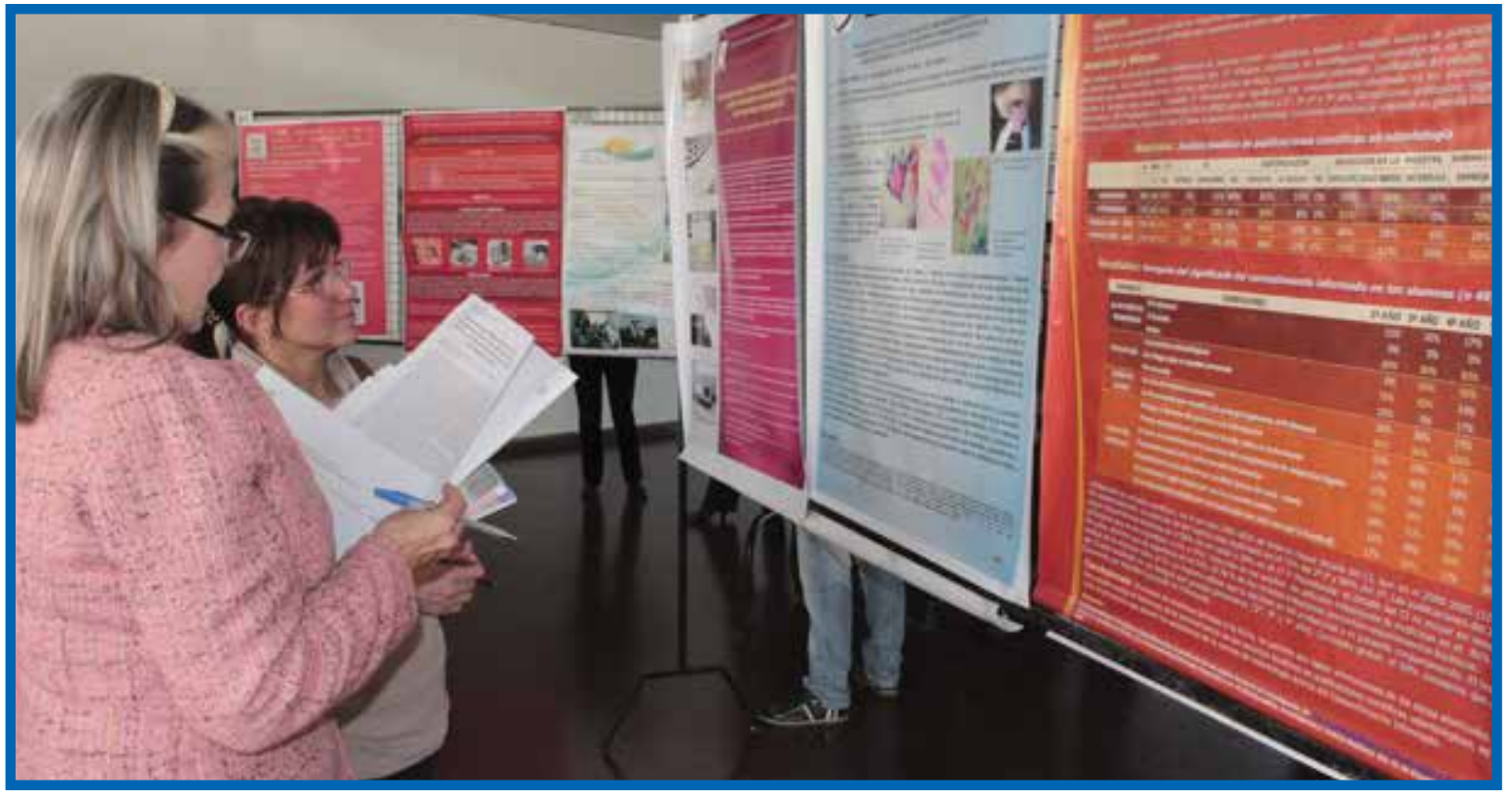

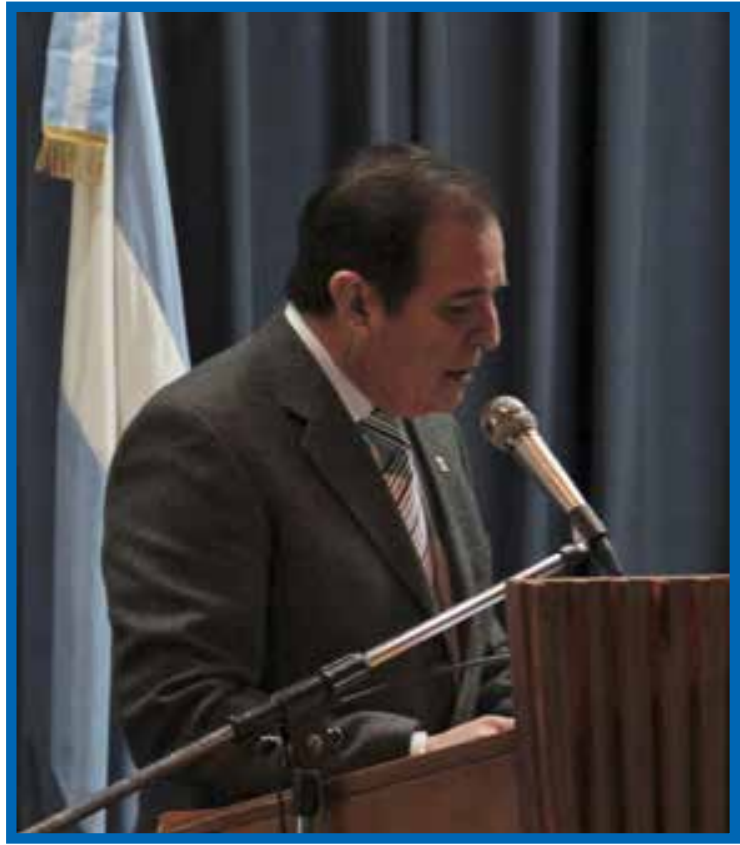

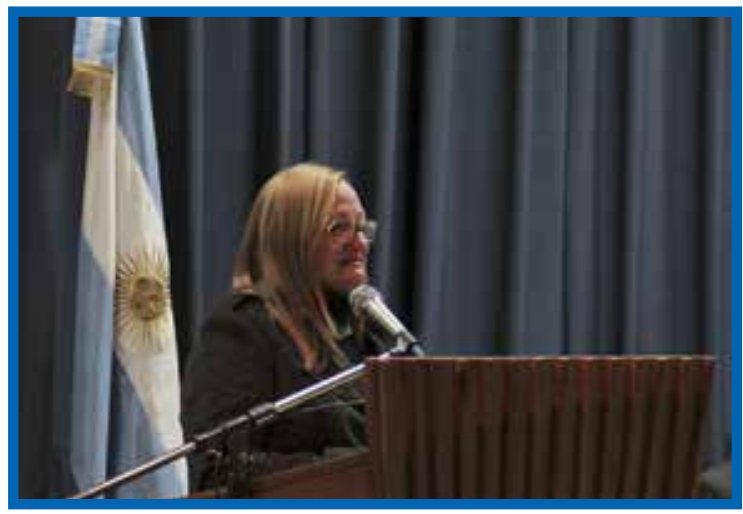

salival en pacientes con periodontitis moderada." Autores: Ledesma Facundo, Acuña Miguel J., Celia Armando, Cuzziol Fernando, Juárez Rolando $P$.

22. "Esmalte y dentina, histomorfologia de la atricion en piezas dentarias temporarias." Autores: Lezcano Melisa R., Gili Maria A., Zamudio Maria A.

23. "Prevalencia de patologías orales en los habitantes naturales del humedal." Autores: Maldonado Seniquel Maria F., Ariasgago Olga L., Ojeda Maria C.

24. "Determinación de la presencia de mesófilas aerobias en los conductos de las unidades dentales." Autores: Morton Natalia S., Ariasgago Olga L., Gallego Maria C., Carzu Oscar R. 25. "Evaluación del Ph Salival en relación al índice de caries en pacientes con discapacidades motoras y sensoriales." Autores: Mosquera Córdoba María C., Vila Vilma G., Barrios Carolina E.

26. "Aspectos bioéticos evidenciados en la publicación científica odontológica." Autores: Marasso Spaciuk Natalia l., Ariasgago Olga L., Rivarola Jose $R$.

27. "Determinación de la presencia de pseudomonas aeruginosa en agua de unidades dentales." Autores: Osnaghi, Mariana l., Gallego, María C., Arzú, Oscar R., Encina Marcela G.

28. "Relación de la vertical pterigoidea con 


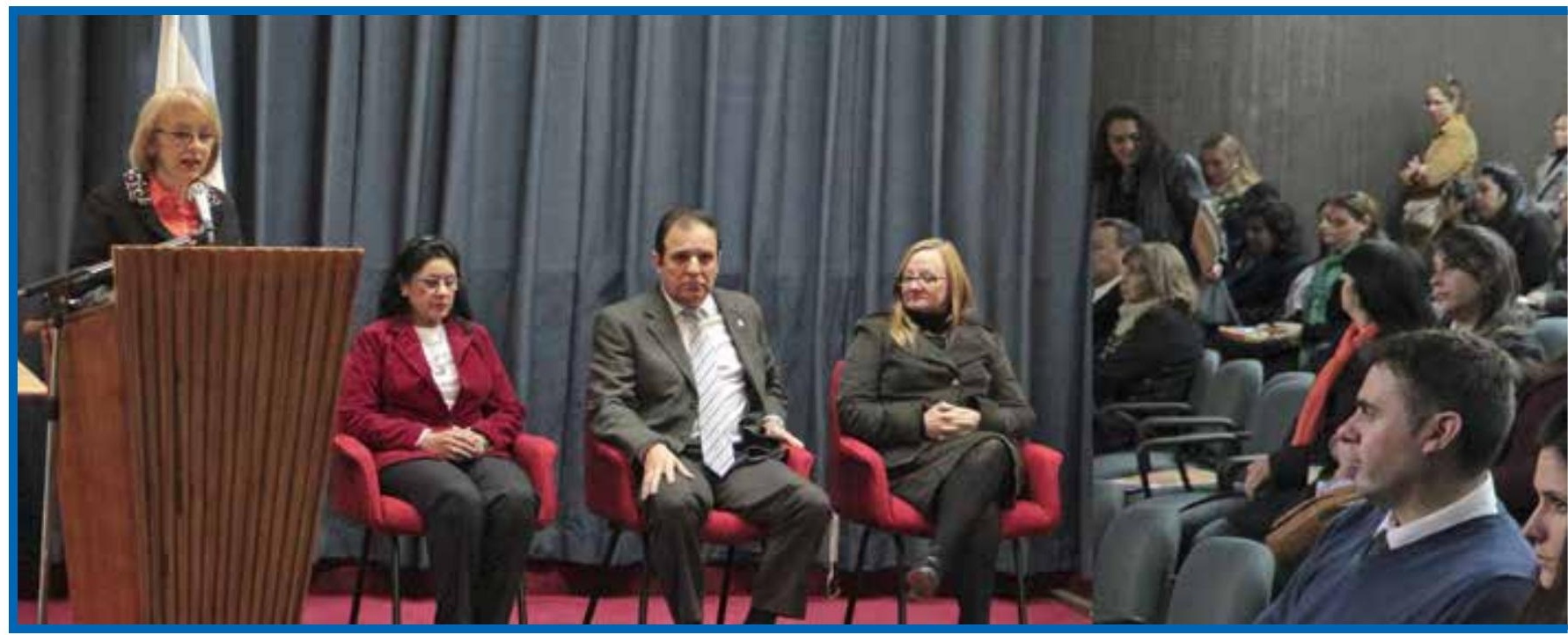

el hueso hioides en pacientes con la convexidad facial reducida." Autores: Palczikowski Lorena Y., Collante de Benítez Carmen I.

29. "Competencias informáticas de los docentes de la Facultad de Odontología de la UNNE." Autores: Panzardi Maria L., Zamudio Maria E., Gallego María C.

30. "Estudio comparativo de tres instrumentos para la toma de citologìa de cavidad bucal en pacientes fumadores crònicos." Autores: Parra Acevedo Rocio S., Almirón Maria S., Juárez Rolando P.

3I. "Cuantificación indirecta de la eliminación del biofilm dental con cepillos manuales y eléctricos, en dentición primaria, mediante fotografía digital." Autores: Perez Quintana Noelia E., Discacciati de Lértora Maria S., Elizondo Maria L.

32. "Frecuencia y distribución de Maloclusión en pacientes adultos jóvenes del Hospital Odontológico de la Facultad de Odontología de la UNNE." Autores: Ponce Alejandro J., Pelaez Alina N., Mazza Silvia M.

33. "Aislamiento e Identificación de Lactobacilos y caracterización del Propóleos." Autores: Ramírez Ayala Claudia N., Lozina Laura, Vaculik Patricia A., Rosende Roque $O$.

34. "Estudio Etnobotánico de Plantas Medicinales para Patologías Buco-Dentales en la región de los Esteros del Iberá." Autores: Rea Ana E., Karaben Viviana E., Zamudio María E.

35. "Utilización de Hierbas Medicinales para el tratamiento de Patologías Bucodentales en la Ciudad de Corrientes." Autores: Redes Florencia, Karaben Viviana E., Morales Sergio $D$.

36. “Expresión de Pcna, Ki-67 Y P53 en Queratoquistesodontogénicos Asociados y no asociados al Síndrome de Carcinoma Nevoide." Autores: Romero María A., González Maria M.

37. "Determinación Clínica de la Clase de Angle en pacientes que concurren al Servicio de Ortodoncia de la Facultad de Odontología UNNE." Autores: Ruiz Díaz Andrea M., Collante de Benítez C., Guiglioni Maria A.

38. "Efectividad de la escala dental de Corah para valorar la ansiedad de los pacientes a anestesia local." Autores: Serrano David O., Ruiz Díaz de Centeno E., Buffil Carlos E.

39. "Primer Screening de Interacciones "In Vitro” de Streptococcus Spp. Y LactobaciIlus Spp. Orales frente a Lactococcus Lactis Subsp. Lactis de origen alimentario." Autores: Sin Cynthya S., Vasek, Olga M., Ortega Silvia M.

40. "Histomorfología del esmalte, dentina y cemento en dientes permanentes con desgaste dental." Autores: Solís Arce Elena P., Zamudio María E., Gili María A.

4I. "Evaluación pronóstica de quiste dentígero con tecnicas inmunohistoquimica." Autores: Sosa Fátima L., Finten Susana B., Briend María S.

42. "Evaluación microbiologica de una for- 


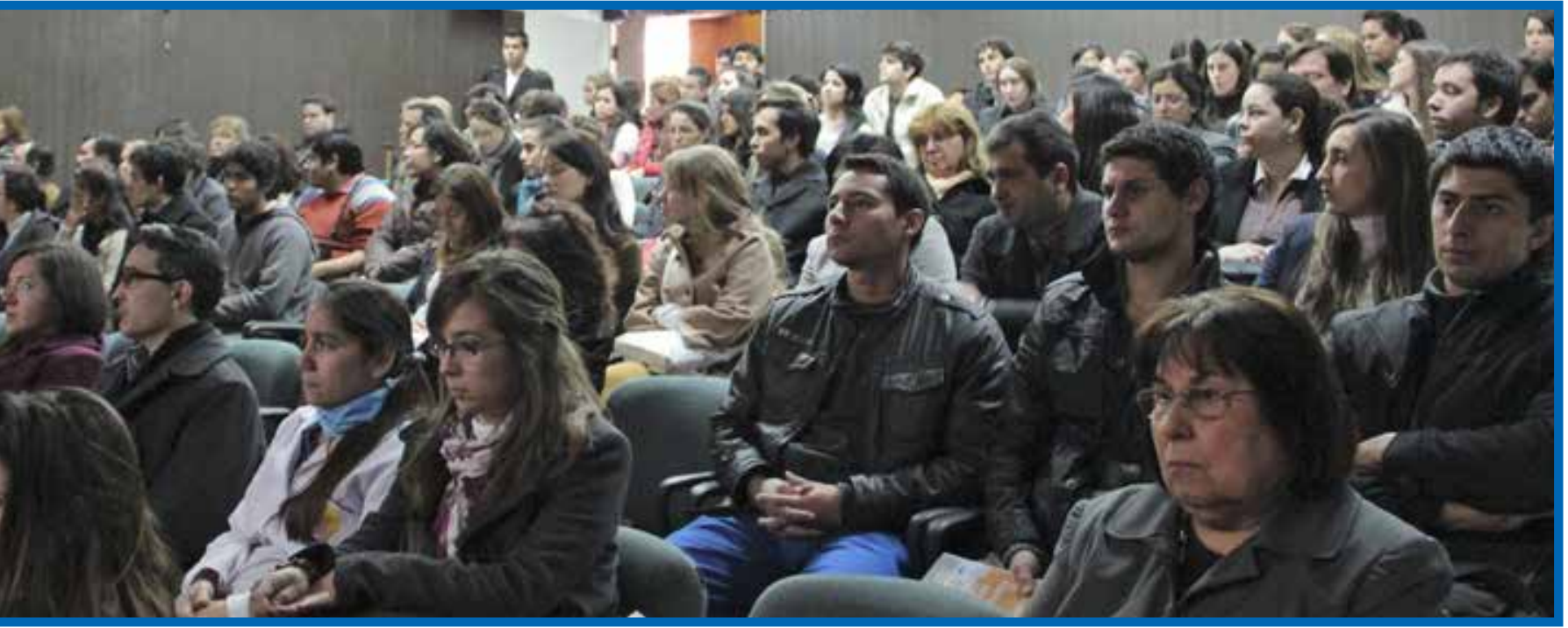

mulacion con propóleos para su uso odontológico.” Autores: Suárez Quinodoz Miguel A., Rosende Roque O.; Lozina Laura A.

43. "Determinación de bisfenol a en saliva humana.” Autores: Quiroz María A., Delfino Mario, Ruiz Díaz de Centeno Elena O., Juárez Rolando P. 44. "Efecto antimicrobiano del propóleos sobre estreptococos sanguinis.” Autores: Hillkirk Wendy, Vaculik Patricia A., Rosende Roque $O$. 45. Aplicación de pruebas paralelas de medición, en sectores laterales de arcos dentarios Inferiores primarios. Autores: Prestia Evelyn G., Galiana Andrea V., Discacciati de Lértora María S.

46 "Determinación de la distribución de tensiones en diferentes Arcos Ortodoncicos". Autores: Farah M., Cabral R.

47. "Efecto de Infusiones de Ilex Paraguariensis sobre la concentración de Fosfatasa Alcalina Salival'”. Autores: Ponce Jorge O., Acuña Miguel J, Celia Armando C, Passetto Roque A, Juárez Rolando $P$.

Se otorgaron premio y menciones especiales a los trabajos presentados por los becarios que fueron entregados en el acto de cierre que se realizó en el Salón Auditorio del Edif. "Prof. Adolfo D. Torres”, presidido por el Sr. Decano quien dio por finalizada las jornadas con un breve discurso expresando su felicitaciones y reiterando la necesidad de continuar trabajando para el avance de la investigación en el ámbito de la facultad, renovando el compromiso de investigadores y directores de los trabajos de investigación.
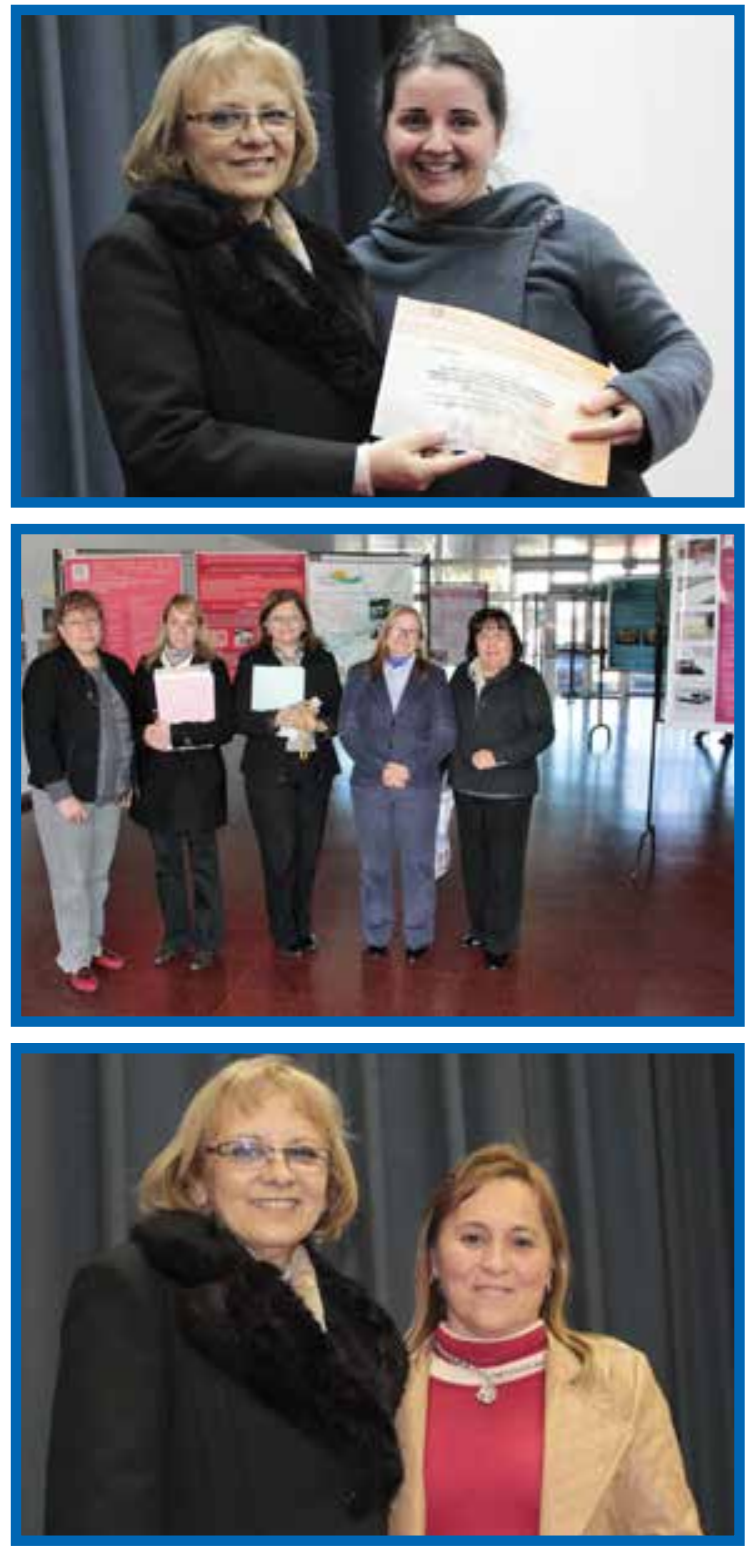